of the most severely affected patients have impaired decision-making skills, they form a minority (Grisso \& Appelbaum, 1995). Most psychiatric patients (including in-patients) are perfectly capable of making decisions regarding treatment and other areas of their lives. It does not help the cause of reducing stigma for our patients to suggest that they cannot make such decisions.

Just as not all psychiatric patients lack capacity, not all medical patients have capacity. This particularly applies to inpatients in whom factors such as cognitive impairment and delirium can affect the ability to make decisions. A recent survey of medical in-patients found that mental incapacity was a very common problem, and one that was frequently overlooked by medical staff (further details available from V.R. upon request). These patients are particularly vulnerable to medical paternalism if this problem is not recognised and appropriately managed.

We agree with Sarkar \& Adshead's call for a code of ethics for British psychiatry, and hope that it will address this difficult area of incapacity. Incidentally, we are also watching with interest the progress of the draft Mental Incapacity Bill. However, we suggest that this area requires careful scrutiny not because psychiatry is a 'special case' but because these issues affect all health care professionals. In this way we could help to lead the way for our non-psychiatric colleagues rather than concentrating on our differences.

Grisso, T. \& Appelbaum, P. S. (1995) The MacArthur Treatment Competence Study. III: Abilities of patients to consent to psychiatric medical treatments. Law and Human Behavior, 19, 149-174.

Sarkar, S. P. \& Adshead, G. (2003) Protecting altruism: a call for a code of ethics in British psychiatry. British Journal of Psychiatry, 183, 95-97.

S. A. Hudson Maudsley Hospital, Denmark Hill, London SE5 8AZ, UK

V. Raymont Institute of Psychiatry, London, UK

\section{Debate on neurosurgery}

The debate on the future of neurosurgery for psychiatric disorders (R. Persaud/ D. Crossley \& C. Freeman, 2003) is curious in many ways. Much of the criticism of neurosurgery still relies upon its historical excesses (Pressman, 1998) rather than the contemporary caution. The 'lack of evidence' argument sets up an unrealistic standard that most surgical treatments are unable to meet. The 'progress in psychiatric treatments' argument fails to recognise that recent drug treatments are but incremental advances over drugs that have been around for some decades, and there are many patients who continue to suffer chronically from depression, obsessive-compulsive disorder and other illnesses. For those of us who practise in tertiary referral centres, encounters with their suffering are frequent and heart-wrenching. Do we wish to take away all their hope?

I am not arguing for a return to the past. Modern neuroscience is fast removing, in a practical sense, the distinction between brain and mind. It is now quite acceptable to consider neural transplants, gene therapy and neural prosthetics as neuropsychiatric treatments. Is this not the right era to revisit surgical interventions on the brain? We are already excited about developments such as vagus nerve stimulation and deep brain stimulation for psychiatric disorders (Malhi \& Sachdev, 2002). We are quite comfortable with ablative surgery for epilepsy when there is functional disturbance, even in the absence of structural abnormality. The neuroanatomical models of psychiatric disorders are becoming increasingly sophisticated (Mayberg, 2001). Should we not be working towards a new era of direct brain intervention, with surgery being an important aspect of this strategy? This surgery may or may not be ablative, or follow an initial period of brain stimulation, or be guided by sophisticated functional imaging. If deep brain stimulation, for example, is demonstrated to produce a therapeutic response without adverse effects, but only temporarily, would there not be an argument to proceed with focal ablation? The brain is, after all, not inviolable, and the evidence is convincing that focal and targeted brain lesions can spare both intellect and personality.

The answer to the question, 'should neurosurgery for mental disorder be allowed to die out?' is surely, 'Definitely not'. Let us, however, move towards a new neurosurgery that is bold but not misinformed, and that keeps abreast of the developments in our understanding of brain function.

Malhi, G. S. \& Sachdev, P. (2002) Novel physical treatments for the management of neuropsychiatric disorders. Journal of Psychosomatic Research, $\mathbf{5 3}$, 709-719.

Mayberg, H. (200I) Depression and frontalsubcortical circuits: focus on prefrontal-limbic interactions. In Frontal-Subcortical Circuits in Psychiatric and Neurological Disorders (eds D. G. Lichter \& J. Cummings), pp. 177-206. New York: Guilford Press.

Persaud, R./Crossley, D. \& Freeman, C. (2003) Should neurosurgery for mental disorder be allowed to die out? (debate). British Journal of Psychiatry, I83, 195-196.

Pressman, J. D. (1998) Last Resort: Psychosurgery and the Limits of Medicine. Cambridge: Cambridge University Press.

P. Sachdev Neuropsychiatric Institute, The Prince of Wales Hospital, Barker Street, Randwick, NSW 203I, Australia

\section{Cognitive-behavioural therapy for psychosis}

Like a magician pulling a rabbit from his hat, Turkington draws a positive result for cognitive therapy for schizophrenia from the literature - only for McKenna to put it back in again (Turkington/ McKenna, 2003). Does it exist or not? McKenna's arguments and table look convincing as, by excluding any study that does not have an active control, he reduces the number of studies he considers. But would he do the same for studies of antipsychotic medications? Or does he assume that patients, and raters evaluating patients, can detect no difference between taking, for example, placebo and haloperidol, or even haloperidol and olanzapine? In which case why are we giving them so much of the latter?

But even focusing only on the studies that he finds acceptable, he dismisses one (SoCRATES; Lewis et al, 2002) for having a positive effect over active control on auditory hallucinations (oh, for a drug that had such an effect over and above those currently available!) and another (Sensky et al, 2000) where a differential benefit of cognitive-behavioral therapy over befriending only became apparent 9 months after therapy ended. He completely omits other widely cited studies with active placebos and positive effects (e.g. Drury et al, 1996). $\mathrm{He}$ then does an unusual meta-analytic exercise in dismissing two small pilot studies by weighing them against each other and finding them to cancel out. Other metaanalyses (e.g. Pilling et al, 2002) using more conventional methodology have concluded differently and, fortunately, so has the National Institute for Clinical Excellence.

The rabbit exists and is multiplying rapidly (e.g. Durham et al, 2003). 